\title{
External magnetic field control of the magnetic breather parameters in a three-layer ferromagnetic structure
}

\author{
E. G. Ekomasov ${ }^{\dagger, 1,2,3}$, V.N. Nazarov ${ }^{4,5}$, A. M. Gumerov' ${ }^{2}$, K. Y. Samsonov ${ }^{1}$, R. R. Murtazin ${ }^{2}$ \\ †EkomasovEG@gmail.com
}

\author{
${ }^{1}$ Tyumen State University, 6 Volodarskogo St., Tyumen, 625003, Russia \\ ${ }^{2}$ Bashkir State University, 32 Zaki Validi St., Ufa, 450076, Russia \\ ${ }^{3}$ South Ural State University (National Research University), 76 Lenin Av., Chelyabinsk, 454080, Russia \\ ${ }^{4}$ Institute of Molecule and Crystal Physics UFRC RAS, 151 Oktyabrya Av., Ufa, 450075, Russia \\ ${ }^{5}$ Bashkir State Medical University, 3 Lenina St., Ufa, 450008, Russia
}

The generation and autoresonant excitation of a magnetic breather in a three-layer ferromagnet by fields of variable frequency and small amplitude in the presence of dissipation in the system is considered. The ferromagnetic structure consists of two wide identical layers separated by a thin layer with modified values of the magnetic anisotropy parameter. The anisotropy parameters are considered functions of the coordinate directed perpendicular to the layer interface. In the one-dimensional case, the function of the anisotropy parameter is modeled in the form of a rectangle. The external magnetic field is variable in time with a small amplitude and frequency, which is a linear function of time. The obtained equation of motion for magnetization in the form of a sine-Gordon equation was solved numerically using an explicit integration scheme. The distribution of magnetization at the initial time was set in the form of a Bloch domain boundary, located far from a thin layer. At certain values of the parameters of a thin layer, when a domain wall passes at a constant speed through it, a magnetic inhomogeneity is formed in the form of a magnetic breather. In the absence of an external field, the breather amplitude decays with time. An analysis of the solutions of the equation of motion in an alternating field shows the possibility, under certain conditions, of increasing with time the amplitude of the magnetic breather. For each case of magnetic anisotropy parameter values, there is a threshold value of the magnetic field amplitude leading to resonance. The resonance effect is also affected by the geometric parameters of a thin layer: with a decrease in the width of the layer, the amplitude of the breather increases more slowly in time. With a large layer width, the translational mode of breather oscillations is also excited.

Keywords: three-layer ferromagnetic, domain walls dynamics, localized magnetic inhomogeneities, sine-Gordon equation, autoresonance.

УДК: 537.611

\section{Управление с помощью внешнего магнитного поля параметрами магнитного бризера в трёхслойной ферромагнитной структуре}

\author{
Екомасов Е. Г. ${ }^{\dagger, 1,2,3}$, Назаров В. Н. ${ }^{4,5}$, Гумеров А. М. ${ }^{2}$, Самсонов К. Ю. ${ }^{1}$, Муртазин Р. Р. ${ }^{2}$ \\ ${ }^{1}$ Тюменский государственный университет, ул. Володарского, 6, Тюмень, 625003, Россия \\ ${ }^{2}$ Башкирский государственный университет, ул. Заки Валиди, 32, Уфа, 450076, Россия \\ ${ }^{3}$ Южно-Уральский государственный университет (национальный исследовательский университет), \\ пр. Ленина, 76, Челябинск, 454080, Россия \\ ${ }^{4}$ Институт физики молекул и кристаллов УФИЦ РАН, пр. Октября, 151, Уфа, 450075, Россия \\ ${ }_{5}^{5}$ Башкирский государственный медицинский университет, ул. Ленина, 3, Уфа, 450008, Россия
}

Рассмотрена генерация и авторезонансное возбуждение магнитного бризера в трехслойном ферромагнетике полями переменной частоты и малой амплитуды при наличии диссипации в системе. Ферромагнитная структура состоит из двух широких одинаковых слоёв, разделённых тонким слоем с изменёнными значениями параметра магнитной анизотропии. Параметры анизотропии считаются функциями от координаты, направленной 
перпендикулярно границе раздела слоёв. В одномерном случае функция параметра анизотропии моделируется в форме прямоугольника. Внешнее магнитное поле является переменным по времени с малой амплитудой и частотой, являющейся линейной функцией времени. Полученное уравнение движения для намагниченности в виде уравнения синус-Гордона решалось численно с использованием явной схемы интегрирования. Распределение намагниченности в начальный момент времени задавалось в виде блоховской доменной границы, находящейся далеко от тонкого слоя. При определенных значениях параметров тонкого слоя при прохождении доменной границы с постоянной скоростью через него, образуется магнитная неоднородность в виде магнитного бризера. В отсутствии внешнего поля амплитуда бризера со временем затухает. Анализ решений уравнения движения в переменном поле показывает возможность при определенных условиях увеличение со временем амплитуды магнитного бризера. Для каждого случая значений параметров магнитной анизотропии имеется пороговое значение амплитуды магнитного поля приводящее к резонансу. На резонансный эффект влияют также геометрические параметры тонкого слоя: при уменьшении ширины слоя рост амплитуды бризера происходит медленнее по времени. При большой ширине слоя возбуждается еще и трансляционная мода колебаний бризера.

Ключевые слова: трёхслойный ферромагнетик, динамика доменных границ, локализованные магнитные неоднородности, уравнение синус-Гордона, авторезонанс.

\section{1. Введение}

Не уменьшается в последнее время интерес к теоретическому исследованию локализованных магнитных неоднородностей солитонного и бризерного типа [1-6]. Это связано с появлением новых экспериментальных методик, позволяющих изучать процессы формирования и распространения локализованных волн намагниченности нанометровых размеров и взаимодействия их с доменными границами (ДГ) [7-12]. Существует в настоящее время программное обеспечение с открытым исходным кодом, решающее зависящие от пространства и времени уравнения Ландау-ЛифшицаГилберта в нано- и микроразмерных ферромагнетиках, например, пакет MuMAX3 [13]. Важным вопросом является нахождение условий генерации устойчивых локализованных волн намагниченности (ЛВН) типа магнитных солитонов и бризеров. Показано ранее, что в области магнитного дефекта $[1,14-16]$ это вполне возможно. Один из надежных способов создания таких магнитных «дефектов» связан с применением многослойных магнитных структур, часто представляющих собой периодически чередующиеся слои двух материалов с различными физическими свойствами [17]. При исследовании динамики линейных и нелинейных волн намагниченности, распространяющихся перпендикулярно слоям часто наличие слоёв, отличающихся друг от друга значением одного или нескольких магнитных параметров, учитывается пространственной модуляцией магнитных параметров материала (см., например, [18 - 20]). В связи со сложностью задачи, даже для одномерного случая, исследователями рассматривалась, как правило, модуляция лишь отдельных параметров магнитной системы. Часто учитывалась, например, модуляция магнитной анизотропии $[15,16,20]$. При определённых условиях, изучение одномерной динамики магнитных неоднородностей приводит к интересной и с математической точки зрения задаче нахождения решения нелинейного дифференциального уравнения типа синус-Гордона с переменными коэффициентами, имеющего важное значение для многих областей современной физики [21].
Для практических применений необходимо изучение способов управления нелинейной динамикой намагниченности в реальном времени. Одним из таких возможных способов управления динамикой намагниченности является приложение внешнего переменного магнитного поля [22] и учет затухания в системе. Известно, что применение авторезонансных моделей управления позволяет существенно уменьшить величину внешнего воздействия на систему [4, 5, 23-29]. Развитие теории управления нелинейной динамической системой в условиях резонанса является так же весьма важной и для многих других технических приложений [30]. Известно, что авторезонанс обнаруживается в колебательных системах различной природы [22-34]. Явление авторезонанса, описываемое нелинейными дифференциальными уравнениями в частных производных (см., например, [35,36]) исследовать достаточно трудно. В работе [37] показано, что микромагнитное моделирование с помощью пакета MuMAX3 может быть успешно применено для моделирования явления авторезонанса при возбуждении спиновых волн в тонких пленках железоитриевых гранатов. Приложения теории авторезонанса к исследованию динамики нелинейных образований в средах с магнитным упорядочением при наличии диссипации и дефектов разработаны пока недостаточно хорошо [22]. Недостатком этих работ является утверждение об изначальном наличии в системе магнитных солитонов и бризеров. В данной статье рассматривается генерация и авторезонансное возбуждение магнитного бризера магнитными полями переменной частоты и малой амплитуды в трехслойном ферромагнетике при наличии диссипации в системе.

\section{2. Основные уравнения и результаты}

Рассмотрим трёхслойную ферромагнитную структуру, состоящую из двух широких одинаковых слоёв, разделённых тонким слоем с изменёнными значениями параметра магнитной анизотропии $[1,2,14]$. Параметры анизотропии считаем теперь функциями от координаты $x$, направленной перпендикулярно границе раздела 
слоёв. Используем для описания динамики намагниченности сферические координаты - $\mathbf{M}(\cos \varphi \sin \theta$, $\sin \varphi, \cos \varphi \cos \theta)$, где $0 \leq \theta \leq 2 \pi-$ угол в плоскости $y z$ между направлением вектора магнитного момента и осью лёгкого намагничивания (ось $O z), \pi / 2<\varphi<\pi / 2-$ угол, описывающий выход $\mathbf{M}$ из плоскости ДГ. Учитывая в плотности энергии магнетика обменное взаимодействие и анизотропию, и считая $\varphi \ll 1$ [17], уравнение движения для намагниченности в угловых переменных можно представить в следующем обезразмеренном виде $[1,2]$ :

$$
\Delta \theta-\ddot{\theta}-\frac{1}{2} f(\vec{r}) \sin 2 \theta=h \sin \theta+\alpha \dot{\theta},
$$

где $f(x)=K(x)=K_{1}(x) / K_{1}^{0}-$ функция, определяющая пространственную модуляцию параметра обменного взаимодействия и константы анизотропии, $K_{1}^{0}$ - константа анизотропии в толстых слоях, $h=\left(H_{Z} / 4 \pi M_{S}\right) Q^{-1}-$ нормированное внешнее магнитное поле, $\alpha=\alpha_{0} / \sqrt{Q}-$ нормированная константа затухания, здесь $Q=K_{1} /\left(2 \pi M_{S}^{2}\right)$ - фактор качества материала, $a_{0}-$ константа затухания. Время $t$ нормировано на $4 \pi M_{s} \gamma \sqrt{Q}$, координата $x$ нормирована на $\delta_{0}$, где $\delta_{0}-$ ширина статической блоховской ДГ. При получении уравнения (1) считалось [1,14], что $K_{1} \ll 2 \pi M_{S}^{2}$, $H_{Z} \ll 4 \pi M_{s}$. Уравнение (1) при нулевой правой части и $K(x)=1$ переходит в известное уравнение синусГордона [11]. Существуют аналитические методы решения этого уравнения [31,38], но, для случая учета произвольных величин внешнего поля, затухания и изменений параметра $K$, необходимо использовать численные методы [39].

Функцию $K(x)$ в одномерном случае для простоты часто моделируют в форме прямоугольника [38]:

$$
f(x)=\left\{\begin{array}{ll}
1, & |x|>W_{x} / 2 \\
K, & |x|<W_{x} / 2
\end{array},\right.
$$

где $W$ - параметр, характеризующий ширину тонкого слоя, $K-$ величина нормированной константы магнитной анизотропии в области тонкого слоя. Будем рассматривать случай тонкого слоя с пониженной по сравнению с широкими слоями величиной магнитной анизотропии. Локализованные высокоамплитудные нелинейные магнитные волны намагниченности типа бризеров возбуждались в области тонкого слоя после прохождения через него движущейся по инерции ДГ аналогично [30]. Амплитуда возбуждаемого магнитного бризера убывает со временем, а его колебания сопровождаются излучением объемных спиновых волн. Для возможных практических применений локализованных магнитных неоднородностей необходимо уметь управлять их структурой и динамическими свойствами. Например, для бризера нужно уметь менять амплитуду и частоту колебаний.

Уравнение (1) решалось численно с использованием метода конечных разностей. Была выбрана трехслойная явная схема решения, с аппроксимацией производных на пятиточечном шаблоне типа «крест» $[16,20,40]$. Данная схема является схемой второго порядка аппроксимации по шагу по координате $\Delta x$ и по шагу по времени $\tau$, обладает условной устойчивостью $(\tau / \Delta x) \leq 1 / 2$. Кроме того, используемая схема удобна тем, что является «одношаговой», использует сравнительно небольшое количество обращений к памяти и обладает потенциалом для оптимизации вычислительного алгоритма. Так же, она с минимальными изменениями может быть адаптирована и для многомерных вариантов уравнения движения (1).

Распределение намагниченности нами в начальный момент времени задавалось в виде блоховской ДГ $\theta_{0}(x)=2 \operatorname{arctg}\left(\mathrm{e}^{x}\right)$, находящейся далеко от тонкого слоя. При прохождении ДГ с постоянной скоростью через тонкий слой образуется магнитная неоднородность в виде магнитного бризера. В отсутствии внешнего магнитного поля амплитуда магнитного бризера со временем затухает.

Для задач управления динамической структурой бризера наиболее интересен случай применения переменного внешнего магнитного поля, когда с помощью резонансных эффектов можно добиться локализованных колебаний намагниченности достаточно большой амплитуды. Внешнее магнитное поле аналогично [24] будем рассматривать как переменное поле вида:

$$
h=h_{0} \cos \omega t
$$

где частота является линейной функцией времени $\omega=\omega_{0}+\mu t$. Здесь $\omega_{0}-$ рассчитанная ранее в $[1,39]$ собственная частота магнитного бризера локализованного в области тонкого магнитного слоя, $\mu$ - малый параметр. Как было показано ранее аналитически [24], с помощью такого вида поля можно ожидать резкого роста амплитуды бризера. Численно подобное явление наблюдалось нами для случая, когда частота внешнего магнитного поля задавалась равной известной собственной частоте локализованных колебаний бризера (см. Рис. 1). Из Рис. 1 видно, что при параметре затухания $\alpha=0.001$ и при начальной частоте колебаний бризера 0.58 за время $t=30$ происходит увеличение в два раза амплитуды магнитного бризера. С уменьшением параметра затухания $(\alpha=0.0001)$ и частоте 0.58 за такое же время происходит увеличение практически в три раза амплитуды магнитного бризера. Здесь следует заметить, что при колебаниях бризер начинает излучать волны, которые при росте амплитуды бризера становятся сильнее. Эти волны приводят к замедлению роста амплитуды бризера, которая выходит на некоторое стационарное значение, не превышающее $2 \mathrm{rad}$.

Рассмотрим теперь влияние амплитуды внешнего магнитного поля. Амплитуда накачки считается малой $h_{0} \ll 1$. Если при $h_{0}=0.1$ имеем случай резонансного увеличения амплитуды бризера (Рис. 1), то при уменьшении амплитуды накачки до $h_{0}=0.05$ происходит небольшой рост амплитуды бризера с дальнейшим ее постепенным понижением при $t>100$ и выходом на стационар с амплитудой около $1 \mathrm{rad}$ (Рис. 2). При малой амплитуде накачки $h_{0}=0.01$ резонансных эффектов не наблюдается, происходит постепенное затухание бризера. Т. е. можно сделать вывод о том, что для каждого случая значений параметров $W$ и $K$ имеется пороговое значение начальной амплитуды магнитного поля, приводящее к резонансу. 


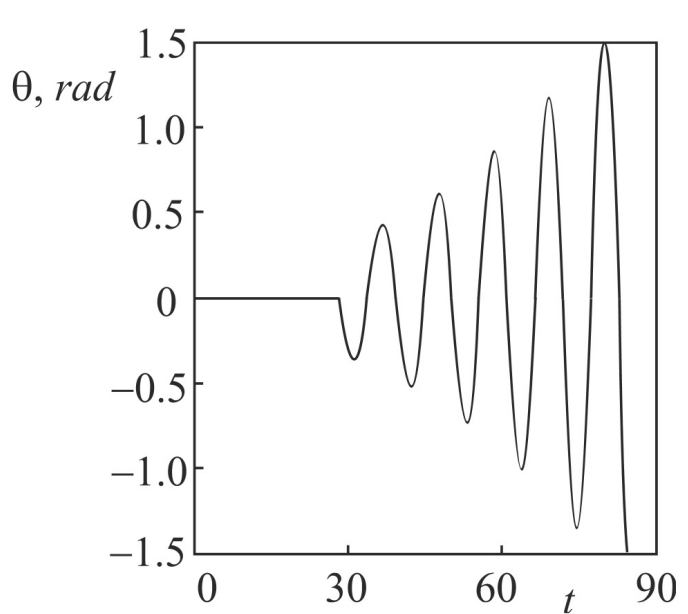

Рис. 1. Зависимость амплитуды колебаний магнитного бризера от времени в переменном поле амплитудой $h_{0}=0.1$. Здесь $W=2$, $K=-0.3$, константа затухания $\alpha=0.001$, частота поля 0.58 , параметр $\mu=0.01$.

Fig. 1. The dependence of the amplitude of the oscillations of the magnetic breather on time in an alternating field with amplitude $h_{0}=0.1$. Here $W=2, K=-0.3$, attenuation constant $\alpha=0.001$, field frequency 0.58 , parameter $\mu=0.01$.

Рассмотрим далее, как влияет на рассмотренный эффект - резонансного увеличения амплитуды магнитного бризера, изменение величины параметров тонкого слоя $W$ и $K$. На Рис. 3 показана зависимость изменения амплитуды бризера от времени для различных значений ширины тонкого слоя. Учтено, что собственная частота магнитного бризера сильно зависит от величины этих параметров [1,39]. Например, для Рис. $3 \mathrm{~b}$ частота 0.82. Найдено, что с увеличением параметра $W$ все быстрее можно (при одном и том же значении величины амплитуды магнитного поля) увеличивать амплитуду бризера. Например, при уменьшении ширины слоя в два раза, рост амплитуды бризера происходит в два раза медленнее по времени. Однако, при ширине слоя $W>2$ не удается раскачать бризер с использованием резонансных эффектов (Рис. $3 \mathrm{a}$ ). При большой ширине дефекта центр колеблющегося бризера не остается в центре слоя, а начинают происходить еще колебательные движения вдоль координаты $x$ (т.е. возбуждается еще и трансляционная мода колебаний бризера).

Аналогичная картина получается с изменением параметра $K$ (Рис. 4). При увеличении по абсолютной величине отрицательного значения параметра $K$ все быстрее происходит рост амплитуды бризера. Но при большом изменении его величины по сравнению c $1(K=-0.5)$ также не удается раскачать амплитуду бризера с использованием резонансных эффектов (Рис. $4 \mathrm{~b}$ ). При большом $K$ колебания бризера хоть и становятся больше, но у излучаемых им волн становится больше амплитуда, что приводит к большей потери энергии у бризера. Только в отличие от предыдущего случая широкого слоя, убыль энергии можно компенсировать увеличением амплитуды магнитного слоя. С уменьшением $K$ все быстрее можно (при одном и том же значении величины амплитуды магнитного поля) увеличивать амплитуду бризера.

Ранее было показано, что магнитную неоднородность бризерного типа можно возбудить в трехслойной ферромагнитной структуре при использовании функции $K(x)$ и другого вида, например, гиперболического [1]. Численные расчеты показали, что в этом случае приложение к магнитному бризеру переменного магнитного поля малой амплитуды специального вида (3) приводит, при определенных условиях, так же к резонансному повышению амплитуды магнитной неоднородности.

\section{3. Заключение}

В работе рассмотрено влияние внешнего переменного магнитного поля на структуру и динамические характеристики магнитных неоднородностей типа бризеров в трехслойном ферромагнетике. Показано, что в переменном магнитном поле специального вида и малой по величине амплитуды можно резонансно повысить амплитуду магнитной неоднородности

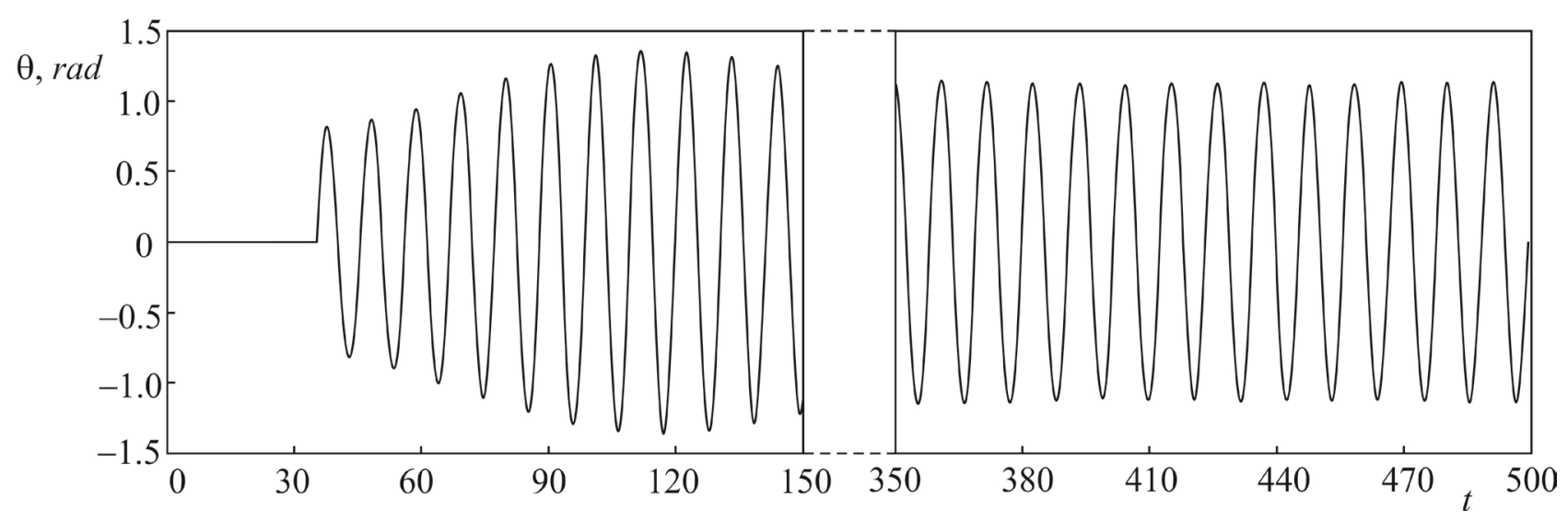

Рис. 2. Зависимость амплитуды колебаний магнитного бризера от времени в переменном поле амплитудой $h_{0}=0.5$. 3десь $\alpha=0.001$, $W=2, K=-0.3$, частота поля $0.58, \mu=0.01$.

Fig. 2. The dependence of the amplitude of the oscillations of the magnetic breather on time in an alternating field with amplitude $h_{0}=0.5$. Here $\alpha=0.001, W=2, K=-0.3$, field frequency $0.58, \mu=0.01$. 

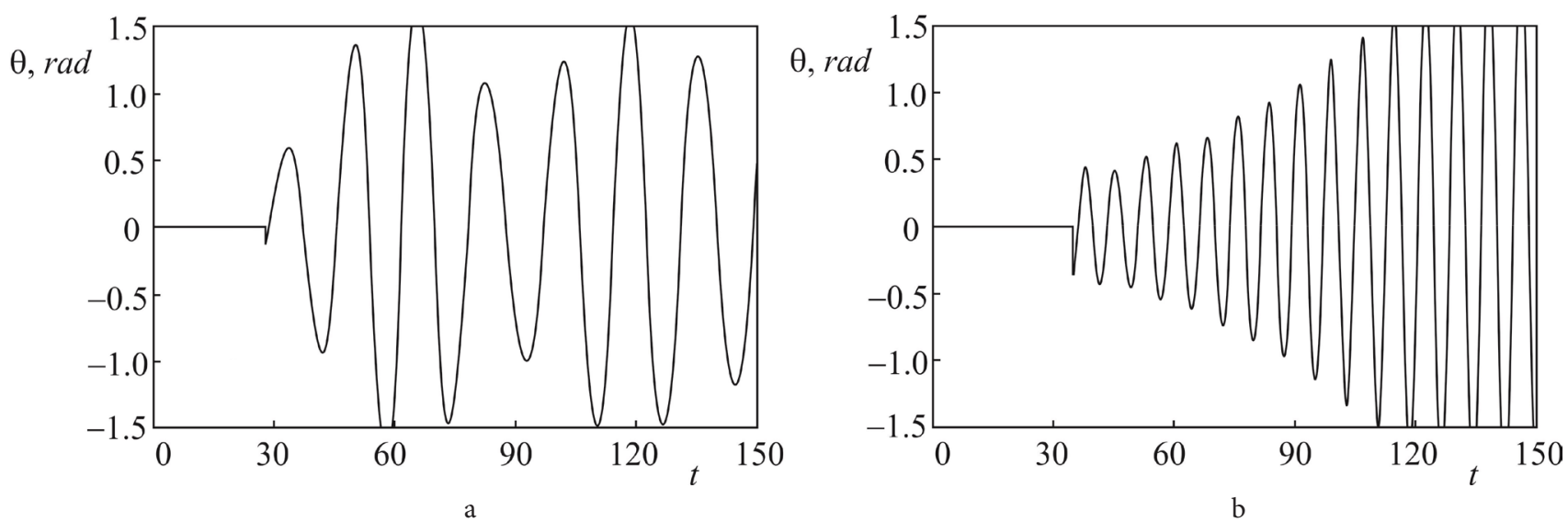

Рис. 3. Зависимость амплитуды колебаний бризера от времени в переменном поле амплитуды $h_{0}=0.1$ при различной ширине слоя: $W=3$ (a), $W=1$ (b). Здесь $\alpha=0.001, K=-0.3, \mu=0.01$.

Fig. 3. Dependence of the breather amplitude of the oscillations on time in an alternating amplitude field $h_{0}=0.1$ for various layer widths: $W=3(\mathrm{a}), W=1$ (b). Here $\alpha=0.001, K=-0.3, \mu=0.01$.
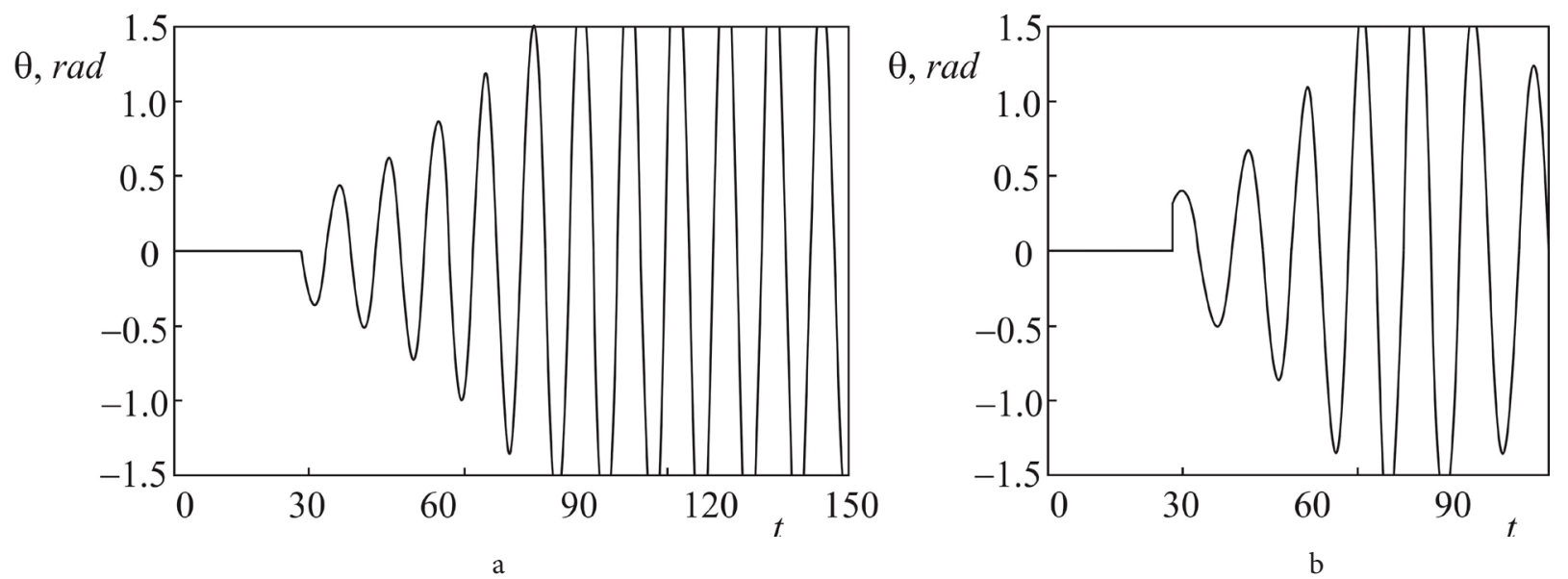

Рис. 4. Зависимость амплитуды колебаний бризера от времени в переменном поле амплитуды $h_{0}=0.1$ при различной глубине слоя: $K=-0.3$ (a), $K=-0.5$ (b). Здесь $\alpha=0.001, W=2, \mu=0.01$.

Fig. 4. Dependence of the breather amplitude of the oscillations on time in an alternating amplitude field $h_{0}=0.1$ at various layer depths: $K=-0.3$ (a), $K=-0.5$ (b). Here $\alpha=0.001, W=2, \mu=0.01$.

бризерного типа. С помощью изменения параметров магнитных слоев можно увеличивать или уменьшать амплитуду и время, необходимое для ее значительного роста. Для каждого набора параметров магнитных слоев существует величина пороговой амплитуды поля, приводящей к авторезонансному росту амплитуды магнитного бризера.

Благодарность/Acknowledgement. Статья выполнена при поддержке Правительства РФ (Постановление №211 om 16.03.2013 г.), соглашение №02.A03.21.0011. Для Гумерова А. М. работа поддержана грантом РФФИ (проект №18-31-00122). Работа выполнена в рамках госзадания \#АААA-A19-119022290052-9. / This article was supported by the Government of the Russian Federation (Decree No. 211 of March 16, 2013), agreement No. 02.A03.21.0011. For Gumerov A. M. this work was supported by a grant from the Russian Foundation for Basic Research (project $\backslash N o \backslash 18-31-00122)$. This work was performed within the state assignment no. \#AAAA-A19-119022290052-9.

\section{Литература / References}

1. E. G. Ekomasov, R. R. Murtazin, V.N. Nazarov. JMMM. 385, 217 (2015). Crossref

2. E. G. Ekomasov, A.M. Gumerov, R.V. Kudryavtsev. Letters on materials. 6 (2), 138 (2016). (in Russian) [Е. Г. Екомасов, А. М. Гумеров, Р. В. Кудрявцев. Письма о материалах. 6 (2), 138 (2016).] Crossref

3. V. V. Kiselev, A. A. Rascovalov. Chaos, Solitons \& Fractals. 45, 1551 (2012). Crossref

4. S. V. Batalov, A. G. Shagalov. Phys. Metals Metallogr. 114 (2), 103 (2013). Crossref

5. S. V. Batalov, V.V. Kiselev, A.A. Raskovalov. Comput. Math. and Math. Phys. 59 (8), 1324 (2019). Crossref

6. P. J. Metaxas, M. Albert, S. Lequeux, V. Cros, J. Grollier, P. Bortolotti, A. Anane, H. Fangohr. Phys. Rev. B. 93, 054414 (2016). Crossref

7. K.S. Novoselov, S.V. Dubonos, S.V. Morozov, D. V. D. Bergen, J. K. Maan, A. K. Geim. Int. J. Nanosci. 3, 87 (2004). $\underline{\text { Crossref }}$ 
8. K.S. Novoselov, A.K. Geim, S.V. Dubonos, E. W. Hill, I. V. Grigorieva. Nature. 426, 812 (2003). Crossref

9. R. Kukreja, S. Bonetti, Z. Chen, D. Backes, Y. Acremann, J.A. Katine, A.D. Kent, H.A. Dürr, H. Ohldag, J. Stöhr. Phys. Rev. Lett. 115, 096601 (2015). Crossref

10. J. P. Tetienne, T. Hingant, J. V. Kim, L. H. Diez, J. P. Adam, K. Garcia, J. F. Roch, S. Rohart, A. Thiaville, D. Ravelosona, V. Jacques. Science. 344, 1366 (2014). $\underline{\text { Crossref }}$

11. M. V. Gerasimov, M. V. Logunov, A. V. Spirin, Yu. N. Nozdrin, I.D. Tokman. Phys. Rev. B. 94, 014434 (2016). Crossref

12. D. Backes, F. Macià, S. Bonetti, R. Kukreja, H. Ohldag, A. D. Kent. Phys. Rev. Lett. 115, 127205 (2015). Crossref

13. J. Leliaert, J. Mulkers. J. Appl. Phys. 125, 180901 (2019). Crossref

14. E.G. Ekomasov, A.M. Gumerov, R.R. Murtazin, R. V. Kudryavtsev, A.E. Ekomasov, N.N. Abakumova. Solid state phenomena. 233-234, 51 (2015). Crossref

15. A.M. Gumerov, E.G. Ekomasov, R.V. Kudryavtsev, M. I. Fakhretdinov. Letters on Materials. 8 (3), 299 (2018). Crossref

16. E.G. Ekomasov, R.R. Murtazin, O.B. Bogomazova, A. M. Gumerov. JMMM. 339. 133 (2013). Crossref

17. D.D. Tang, Y.-J. Le. Magnetic Memory: Fundamentals and Technolog. Cambridge University Press, New York (2010) 196 p. ․ㅡossref

18. V.S. Tkachenko, V.V. Kruglyak, A.N. Kuchko. Metamaterials. 3, 28 - 32 (2009). Crossref

19. E. Della Torre, C. M. Perlov. J. Appl. Phys. 69, 4596 (1991). Crossref

20. E.G. Ekomasov, Sh.A. Azamatov, R.R. Murtazin. Phys. Met. Metallogr. 105 (4), 313 (2008). Crossref

21. The Sine-Gordon Model and Its Applications: From Pendula and Josephson Junctions to Gravity and High-energy Physics. Vol. 10 (Ed. by J. Cuevas-Maraver, P.G. Kevrekidis, F. Williams). Springer (2014) 263 p. Crossref

22. V. N. Nazarov, L. A. Kalyakin, M. A. Shamsutdinov. Solid State Phenomena. 168-169, 81 (2011). $\underline{\text { Crossref }}$

23. L. A. Kalyakin. J. Math. Sci. 125 (5), 658 (2005). Crossref

24. E. G. Ekomasov, V. N. Nazarov. Letters on materials. 8 (2), 158 (2018). (in Russian) [В. Н. Назаров, Е. Г. Екомасов. Письма о материалах. 8 (2), 158 (2018).] ㄷossref
25. L. A. Kalyakin. Russian Math. Surveys. 63 (5), 791 (2008). Crossref

26. R.N. Garifullin, L.A. Kalyakin, M.A. Shamsutdinov. Comput. Math. and Math. Phys. 47, 1158 (2007). Crossref

27. L. A. Kalyakin, M. A. Shamsutdinov. Theor. Math. Phys. 160, 960 (2009). Crossref

28. S. V. Batalov, E. M. Maslov, A. G. Shagalov. J. Exp. Theor. Phys. 108, 890 (2009). Crossref

29. A. I. Neishtadt. Proc. Steklov Inst. Math. 250, 183 (2005). (in Russian) [А.И. Нейштадт. Тр. МИАН. 250, 198 (2005).]

30. A.L. Fradkov. Phys. Usp. 48, 103 (2005). Crossref

31. B. Meerson, L. Friedland. Phys. Rev. A. 41 (9), 5233 (1990). Crossref

32. J. Fajans, L. Friedland. Am. J. Phys. 69 (10), 1096 (2001). Crossref

33. L.A. Kalyakin, O.A. Sultanov, M.A. Shamsutdinov. Theoret. and Math. Phys. 167 (3), 762 (2011). Crossref

34. L. A. Kalyakin. Theoret. and Math. Phys. 194 (3), 331 (2018). Crossref

35. L. Friedland, A. G. Shagalov. Phys. Rev. E. 71, 036206 (2005). Crossref

36. L. Friedland, A. G. Shagalov. Phys. Rev. E. 73, 0666122006 (2006). Crossref

37. V.S. Teplov, V.D. Bessonov, S. V. Batalov, A. V. Telegin. J. Phys.: Conf. Ser. 1389, 012141 (2019). Crossref

38. M. A. Shamsutdinov, I. Yu. Lomakina, V.N. Nazarov, A.T. Kharisov, D.M. Shamsutdinov. Ferro- i antiferromagnitodinamika. Nelineynyye kolebaniya, volny i solitony. Moscow, Nauka (2009) 456 p. (in Russian) [М.А. Шамсутдинов, И.Ю. Ломакина, В.Н. Назаров, А.Т. Харисов, Д.М. Шамсутдинов. Ферро- и антиферромагнитодинамика. Нелинейные колебания, волны и солитоны. Москва, Наука (2009). 456 с.]

39. E. G. Ekomasov, R. R. Murtazin, S. A. Azamatov. Phys. Solid State. 54 (8). 1584 (2012). Crossref

40. A.M. Gumerov, E.G. Ekomasov, R.R. Murtazin, V.N. Nazarov. Comput. Math. Math. Phys. 55 (4), 628 (2015). . Crossref 